\title{
The influence of food restriction on the small bowel: Does intensive short-term food restriction lead to weight loss?
}

\author{
Makovicky $\mathrm{P}^{1,2}$, Tumova $\mathrm{E}^{3}$, Volek $\mathrm{Z}^{4}$, Makovicky $\mathrm{P}^{5}$, Arnone $\mathrm{JM}^{6}$, Svecova ${ }^{1,2}$, Samasca $\mathrm{G}^{7}$
}

Czech Centre for Phenogenomics, Division BIOCEV, Vestec, Czech Republic. pmakovicky@email.cz

\begin{abstract}
The objective of this study was to show morphological changes in the small intestine of Hyplus broiler rabbits following an eight-day food restriction programme. The control group (C) received food ad libitum (ADL) for the duration of the experiment. Group R1 received $50 \mathrm{~g}$ of food per day, and group R2 received $65 \mathrm{~g}$ of food per day. After the food restriction diet had been completed, groups R1 and R2 were returned to ad libitum feeding. After food restriction and at the end of the experiment, the longest small bowel measurement was recorded in the C group. In the $\mathrm{C}$ group, after food restriction, the villi height was significantly higher, compared to that in R1 and R2 groups and at the end of the experiment, the villi were significantly higher in R1 and R2 groups. After food restriction, the values of crypts depth were approximately similar in all groups, and the end of experiment, the depth of crypts were deepest in R1and R2 groups, as compared to that in C group. The full process is followed by weight loss to the end of the experiment. These data suggest that intensive short-term food restriction followed by ADL feeding has effect on weight loss (Fig. 3, Ref. 30). Text in PDF www.elis.sk.

KEY WORDS: experimental medicine, food restriction, rabbit, slimming, small bowel, weight loss.
\end{abstract}

\section{Introduction}

Food restriction is a well-known method for weight loss. It is also known as one of the oldest methods of treating and preventing some diseases in humans as well as animals $(1,2,3)$. For example, Claudius Galen recommended this method for overweight patients (4). Intensive development of applied medicine and science relating to the effects of food restriction has resulted in identifying numerous aspects related to food restriction, but there are still aspects that remain unclear. The small bowel has several tasks, with the main one being nutrient resorption $(5,6)$. Quantitative differences in small bowel resorptions can also affect other organ

${ }^{1}$ Czech Centre for Phenogenomics, Division BIOCEV, Vestec, Czech Republic, ${ }^{2}$ Department of Transgenic Models of Diseases, Institute of Molecular Genetics, AS CR v.v.i., Prague, Czech Republic, ${ }^{3}$ Department of Animal Husbandry, Faculty of Agrobiology, Food and Natural Resources, Czech University of Life Sciences in Prague, Czech Republic, ${ }^{4}$ Physiology of Nutrition and Quality of Animal Product, Institute of Animal Science in Prague-Uhrineves, Czech Republic, ${ }^{5}$ Department of Biology, Faculty of Education, Selye Janos University, Komarno, Slovak Republic, ${ }^{6}$ School of Nursing, Kean University, Union New Jersey, U.S., and ${ }^{7}$ Department of Immunology, Iuliu Hatieganu University of Medicine and Pharmacy, Cluj-Napoca, Romania

Address for correspondence: P. Makovicky, Ing, PhD, Czech Centre for Phenogenomics, Institute of Molecular Genetics AS CR v.v.i., Division BIOCEV, Prumyslova 595, CZ-252 50 Vestec, Czech Republic. Phone: $+420776842684$

Acknowledgement: The work was institutionally supported by RVO 68378050, LM2015040 (MEYS) and project BIOCEV - Biotechnology and Biomedicine Centre of the Academy of Sciences and Charles University (CZ.1.05/1.1.00/02.0109; European Regional Development Fund) and by NAAR QJ15100192. systems and have an important impact on health. It was found that the changes in length of the small bowel, height of villi and depth of crypts are dependent on the intensity of food restriction. It has also been shown that after food restriction with subsequent transition to ad libitum (ADL) feeding, there is a lengthening of the small bowel compared to the control group (7). These results were documented on limited materials and currently it is not clear how food restriction affects the entire small bowel. It is an important question, which can clarify some other open questions. Mainly it is about the integrity of the adaptation of individual anatomical parts of the small bowel to the duration and intensity of food restriction. Subsequently it is also about adaptation to the postrestriction period with $\mathrm{ADL}$ intake and some related changes in the small bowel morphology. Such knowledge may provide answers to the question whether intensive short-term restriction is an appropriate approach to weight loss. We hypothesize that after intensive short-term food restriction with subsequent ADL feeding, the height of villi is going to grow intensively, thus increase the intestinal absorptive area and result in excessive weight gain. These findings can be very important for clinical praxis because they are related to the general use of food restriction for weight loss. It is not clear which anatomical part of the small bowel is more sensitive to food restriction and how they subsequently react to the resumption of ADL intake following food restriction. These questions can be important for physiological research and they can also find utility in studies dealing with gastrointestinal resorption. The objective of our work is to perform histological analyses of the small bowel under intensive short-term food restriction, including the degree of variability in the percentage of food restriction in combination with ADL intake in an experimental rabbit model. 


\section{1-365}

\section{Material and methods}

Animal ethics.

This study was approved by the Ethics Committee of the Institute of Animal Science and the Central Commission for Animal Welfare of the Ministry of Agriculture of the Czech Republic and was carried out in accordance with the guidelines for applied nutrition experiments in rabbits (8).

\section{Study design}

Small bowel samples from broiler rabbits kept in special experimental conditions were analysed. The experiment with Hyplus broiler rabbits was conducted in the rabbit building of the Institute of Animal Science in Uhrineves in Czech Republic from a weaning age of 32 days up to 70 days of age. All rabbits were kept under controlled environmental conditions and housed in standard cages. They were divided into three groups. The control group was fed ADL during the entire experiment (C). The first experimental group was restricted between 35 and 42 days of age, when the rabbits received $50 \mathrm{~g}$ of food per rabbit per day (R1). The second experimental group was also restricted between 35 and 42 days of age, but the rabbits obtained $65 \mathrm{~g}$ per rabbit per day (R2). Before and after restriction, all rabbits were fed ADL with a commercial food mixture. The design of the experiment is shown in Figure 1.

\section{Sample collection}

For small bowel analysis, eight rabbits per group were weighed and then slaughtered at 42, 49 and 70 days of age, from all three (C, R1, R2) groups. The length of the entire small intestine, from pylorus to ileocaecal junction, was measured and then three tissue samples were obtained from each rabbit, namely from

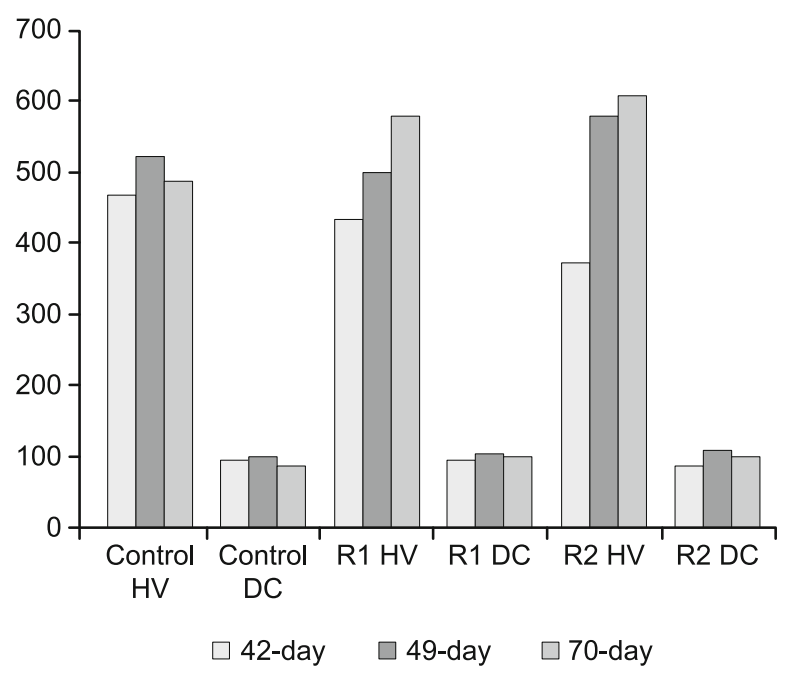

Fig. 1. The height of villi and depth of crypt in control, R1 and R2 groups. $\mathrm{R} 1$ - restriction $50 \mathrm{~g}$ of food per rabbit/per day, $\mathrm{R} 2$ - restriction $65 \mathrm{~g}$ of food per rabbit/per day, HV - height of villi, DC - depth of crypts. the duodenum, jejunum and ileum. All samples were collected within 30 minutes after slaughtering and from the same anatomical place. Small bowel samples were fixed with a $4 \%$ Bouin solution for two days.

\section{Sample evaluation and processing}

Samples were processed by standard histological methods and then embedded in paraffin blocks. The slices were stained with haematoxylin-eosin (DiaPath, Italy). The height of the villi and the depth of the crypts were measured using the NIS-Elements AR version 3.0 software (Laboratory Imaging s.r.o., Czech Republic). The height of villi was measured from the top of the villus to the crypt transition, and the crypt depth was defined and measured as the invagination between two villi. The heights of 50 villi, and the depths of 50 crypts were individually measured per each duodenum, jejunum and ileum sample.

\section{Statistical analysis}

The results were evaluated using the SAS program (9), and the ANOVA method $(\mathrm{p}<0.05)$.

\section{Results}

The results of weight, length and measurements of villi heights and crypt depths are shown in Figure 2. All morphometric measurements, segregated according to the different parts of the small bowel, are shown in Figure 3.

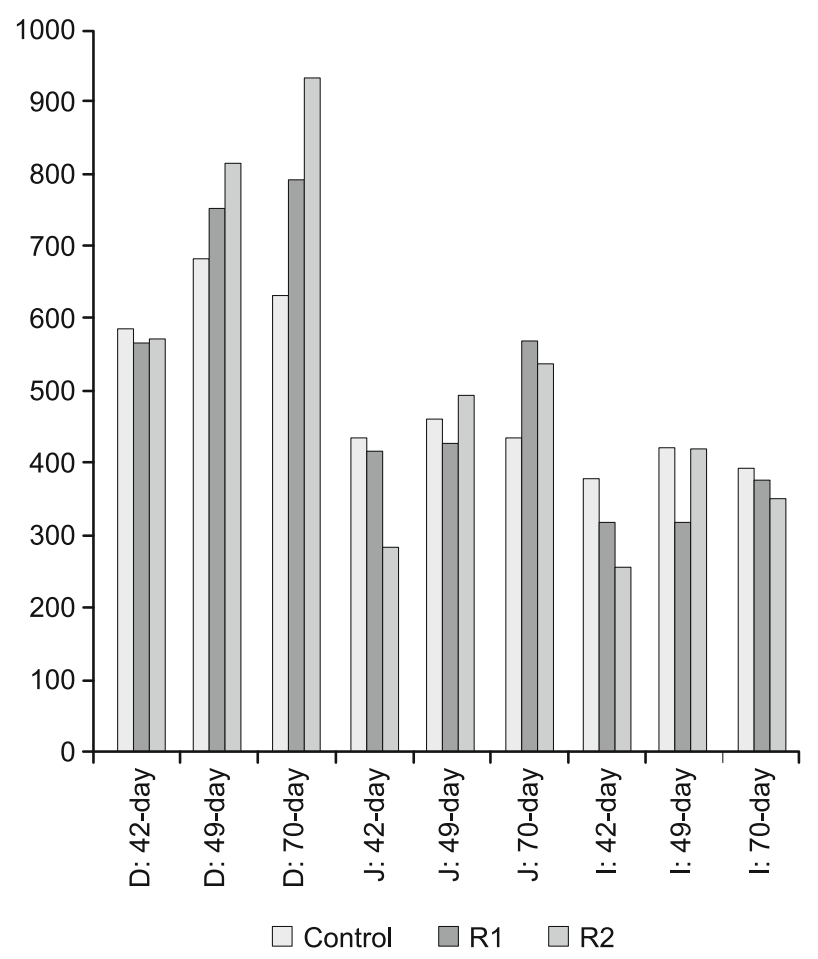

Fig. 2. The height of villi in different part of small bowel. R1 - restriction $50 \mathrm{~g}$ of food per rabbit/per day, R2 - restriction $65 \mathrm{~g}$ of food per rabbit/per day, D - duodenum, J - jejunum, I - ileum. 


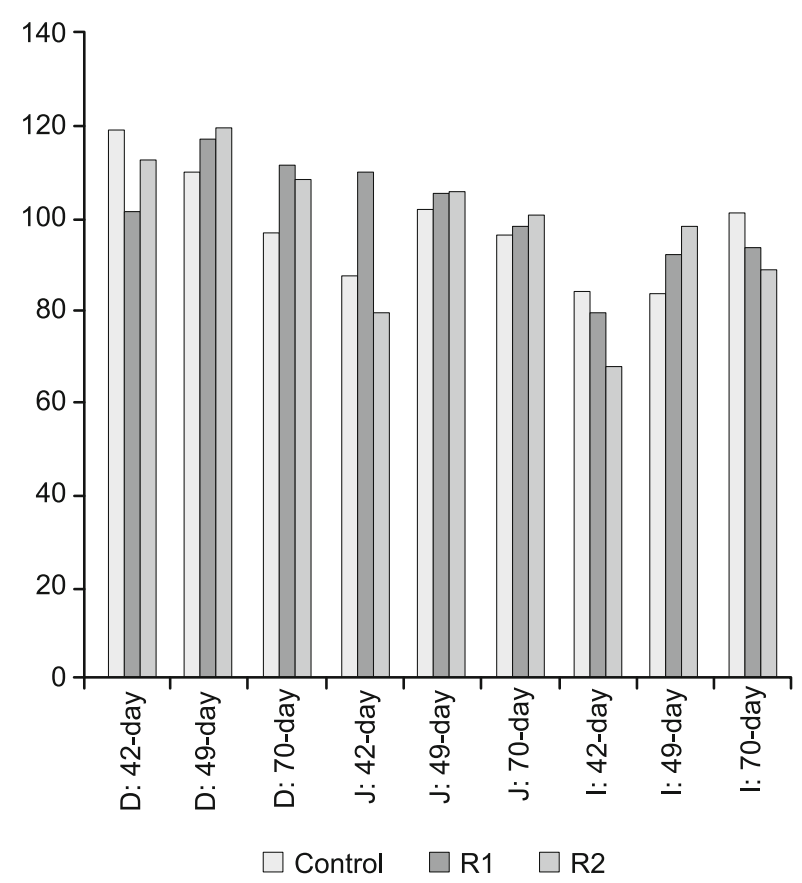

Fig. 3. The depth of crypt in different part of the small bowel. R1 - restriction $50 \mathrm{~g}$ of food per rabbit/per day, R2 - restriction $65 \mathrm{~g}$ of food per rabbit/per day, D - duodenum, J - jejunum, I - ileum.

Weight of rabbits

After restriction, there were some differences between rabbits' weights, with the heaviest average weight measured in the $\mathrm{C}$ group. Almost continuous weight gain occurred in all groups with the maximum weight recorded at the end of experiment for all groups when some differences were visible $(\mathrm{p}<0.05)$.

\section{Length of the small bowel}

Immediately after restriction at the first sampling the longest small bowel measurement was recorded in the $\mathrm{C}$ group. There was a further increase in the length of the small bowel observed to the end of the experiment.

\section{Villi height}

Results show changes between $\mathrm{C}$ group and $\mathrm{R} 1, \mathrm{R} 2$ groups in some measured parameters. In the $\mathrm{C}$ group, immediately after food restriction on day 42 , the villi were significantly higher, compared to R1 and R2 groups $(p<0.05)$. At the end of the experiment, the situation was opposite, with the villi being significantly higher in $\mathrm{R} 1$ and $\mathrm{R} 2$ groups $(\mathrm{p}<0.05)$. As to the different anatomical parts of the small bowel, immediately after food restriction, a minimal change in duodenal villi height was visible among all three groups. At the end of the experiment, the R1 and R2 groups had a higher duodenal villi height, compared to the $\mathrm{C}$ group $(\mathrm{p}<0.05)$. Immediately after food restriction, jejunum villi were higher in the $\mathrm{C}$ group $(\mathrm{p}<0.05)$. At the end of the experiment the situation changed. The jejunum villi were highest in $\mathrm{R} 1$ and $\mathrm{R} 2$ groups ( $\mathrm{p}$ $<0.05$ ). The morphometric analysis of ileum villi height shows that in all three groups there was gradual villi growth visible, but the ileum villi were highest in the $\mathrm{C}$ group from the start to the end of the experiment.

\section{Crypt depth}

The results show changes between $\mathrm{C}$ group and $\mathrm{R} 1$, $\mathrm{R} 2$ groups in the measured parameters. Immediately after food restriction, the values of crypts depth were approximately similar in all groups, but at the end of experiment, the depth of crypts were deepest in $\mathrm{R} 1$ and R2 groups, as compared to the $\mathrm{C}$ group. The crypt deepening was observed in all three groups after ADL feeding, but at the end of experiment the value was lower compared to ADL feeding immediately after food restriction. At the beginning of the experiment, the duodenal crypts were deeper in the C group, compared to the R1, R2 groups, but at the end of the experiment the situation reversed and the duodenal crypts were deeper in R1and R2 groups. The crypt depth in the jejunum exhibited some changes after food restriction, while at the end of experiment, R1 and R2 group crypts were deeper. From the beginning, and at the end of experiment, the ileum crypts were deeper in the $\mathrm{C}$ group, compared to $\mathrm{R} 1$ and $\mathrm{R} 2$ groups, with minimal differences immediately after ADL feeding in R1 and R2 groups.

\section{Discussion}

Today it is known that variability in diet can modify small bowel morphology, including the height of villi and depth of crypts (10). There is plenty of information in literature about the effects of food restriction on the small bowel. The results of an experiment in rats subjected to a $50 \%$ dietary food restriction showed that there was hypertrophy of the duodenal mucosa and atrophy of the ileal mucosa (11). The numbers of goblet cells and intraepithelial lymphocytes were reduced, but the mitotic index remained unaltered in both the duodenum and ileum. In our experiment with rabbits, we do not find the same histopathological changes in the small bowel. The observed changes in small bowel morphology suggest that intensive short-term food restriction can be beneficial for subsequent, more efficient resorption. This effect is even observed in less intensive food restriction ( $65 \mathrm{~g} /$ per rabbit), and not just in the more stringent food restriction ( $50 \mathrm{~g} / \mathrm{per}$ rabbit). Other groups' findings are in concordance with these observations and have been ascribed to gastrointestinal immunity modulation and gut microbiota $(12,13)$. The work of Tsiouris et al shows that feed restriction of broiler chicks has a positive effect on the intestinal ecosystem and significant protective effect against necrotic enteritis in a subclinical experimental model (14). The results of Knudsen et al show that feed-restricted rabbits tended to have greater expressions of interleukin and lower expressions of tumour necrosis factor- $\alpha$, suggesting that food restriction can modulate gut immunity (15). It has been also shown, that small bowel morphology changes during fasting and refeeding are accompanied by heat shock protein modulation (16). We assume, that there are some influences and dependencies between individual parts of small bowel and food restriction. The duodenal part was minimally affected by intensive short-term food restriction, but on the other hand, it is the most 


\section{1-365}

sensitive part to ADL intake. The jejunum show a less sensitive reaction in the post-restriction period with ADL intake than the duodenum. The ileum part is least affected by the post-restriction period with ADL intake. These results indicate that the duodenum is less affected by short-term food restriction, and more affected by subsequent $\mathrm{ADL}$ feeding, while in the distal direction, the effect of intensive short-term food restriction becomes stronger, but the reaction to subsequent ADL feeding is less intense. So altogether it seems that the proximal part of the small bowel can absorb the most nutrients from the restricted food intake, just as well as from ADL food intake. The results of Tumova et al shows that a one-week feed restriction can modify the morphology of intestinal tract of a growing rabbit (17). At the end of this experiment, the dietary-restricted rabbit group had the longest small bowel, highest villi and deepest crypts, compared to ADL-fed rabbits. Our work has deepened these findings, as we further analysed the different parts of the small bowel. Our results show that short-term food restriction has a potential beneficial effect for health, because there is no decrease in villi height that has been generally associated with reduced intestinal health. In the experiment of De Oliveira et al, dietary restrictions from 33 to 40 days of age and from 54 to 61 days of age, were associated with no effects on duodenal morphometry (18). In the jejunum, the villi of ADL-fed rabbits or of those with diet restricted from 33 to 40 days were higher, and rabbits with diet restricted from 33 to 40 days had wider villi and higher absorption surface. In the ileum, rabbits fed ADL had higher villi. The work also showed that feed restriction reduced the heart weight, but not its size, and negatively affected jejunum morphometry when performed in later stages. At the same time, literature contains conflicting data indicating that feed restriction does not significantly influence performance indices, carcass yield, organ weights and body dimensions (19). Another work shows that in addition to decreasing the enzymatic activity in the enterocytes, the feed restriction can result in lower villus perimeter and lower crypt depth, (20). In our previous work, we have shown that feed restriction in rabbits can affect small bowel morphology in the duodenum (21). In our first work dealing with food restriction applied on rabbits between 42 and 49 days, significant interactions $(\mathrm{p}<0.05)$ between group and age were documented as to villi height and crypt depths of duodenum (22). These findings support the theory that villus height and crypt depth and length of the small intestine change with the intensity of feed restriction and age. Considering intensive short-term food restriction and weight loss, there is a gap in current knowledge. It is assumed that dietary food restriction prevents excessive body weight gain (23). For a long time, researchers have searched for appropriate overweight and obesity treating tools. Long-term food restriction looks like one possibility to reduce weight. Restricted feeding, and thus limited caloric intake, prevents many health problems and increases animal lifespan (24). It is also a recommended method in human clinical practice. Nevertheless, a question should be posed as to whether intensive short-term food restriction followed by ADL feeding is a really good method for weight loss. In our experiments, we have demonstrated that immediately after intensive short-term food restriction, followed by ADL feeding, there were increases in the length of villi in the small bowel. Our results documented that immediately after food restriction, the height of villi in R1 and R2 groups were smaller, compared to the $\mathrm{C}$ group. After a short ADL feeding period, there was an intensive growth period and the villi were highest in $\mathrm{R} 1$ and $\mathrm{R} 2$ groups, compared to the $\mathrm{C}$ group. This intensive growth was subsequently reflected with an increase in absorption surface, thus leading to a better use of nutrients by the body. Immediately after food restriction, the weight of rabbits in $\mathrm{R} 1$ and R2 groups were lower, compared to the C group, while the same results were observed at the end of experiment. Full period was accompanied by increasing height differences. At 42 days of age there were some weight differences between C and R1 ( $-43 \mathrm{~g})$; $\mathrm{C}$ and R2 ( $-32 \mathrm{~g})$. At 49 days of age the differences were greater, namely between C and R1 (-131 g); C and R2 ( $-40 \mathrm{~g})$ while at the end of experiment they were as follows: $\mathrm{C}$ and R1 ( $-343 \mathrm{~g})$; $\mathrm{C}$ and $\mathrm{R} 2(-380 \mathrm{~g})$. The results showed, that an intensive short-term food restriction is an appropriate instrument for gastrointestinal tract recovery and has effects on weight loss. Other references showed, that a more effective instrument for weight loss is the intermittent fasting or alternate day fasting. It is clear, that it is not a universal medicine and also that one approach does not fit all in the quest for achieving body weight control, but this dietary strategy could be considered as an option for achieving weight loss and its maintenance (25). The findings of Zhang et al show that simply reducing the amount of food intake results in an increased appetite accompanied with obvious weight regain, and suggested that the resulting enlargement of villi surface area plays a key role in the regain of weight (26). The experimental work of Dou et al described an intensive 7-day fasting on a rat model and showed that the rats regained the lost body weight ( $22 \%$ ) by the 7 th day of re-feeding (27). The lost duodenal mass ( $40 \%$ ) and jejunum mass (25\%) were regained by the 2 nd day whereas the lost mass from the ileum (18 $\%$ ) was regained by the 4 th day. The fasting-induced morphometric changes were normalised by re-feeding on the 2 nd day in the duodenum and jejunum, and on the 4 th day in the ileum. The results of another important work dealing with the influences of short 2-day fasting periods, show significantly decreased iron concentrations in serum and hair, as well as levels of ferritin, haemoglobin, haematocrit, red blood cells, and total iron binding capacity, however this short-term fasting did not influence the other iron management parameters (28). These changes can alter the emotional status of slimming women and can be in association with depression. This may be a global worldwide problem because many women are discontented with their appearance, including weight $(29,30)$. They indicate that some restrictive diets can be finally harmful, but most of them still stay ineffective following weight gain. The results of our work explain that weight loss based on intensive short-term food restriction, when followed by ADL feeding, is compensated by improved nutrient resorption due to the expansion of small bowel resorption capacity, and has effects on weight loss.

\section{Conclusion}

This work confirms, that an intensive short-term food restriction influences all three parts of the small bowel. Intensive short-term food restriction followed by ADL feeding has effects on weight loss. 


\section{References}

1. Ginter E. Vegetarian diets, chronic diseases and longevity. Bratisl Lek Listy 2008; 109 (10): 463- 466.

2. Noyan H, El-Mounayri O, Isserlin R et al. Cardioprotective signature of short-term caloric restriction. PLoS One 2015 10: e0130658.

3. Pastorelli H, Le Floch N, Merlot E, Meunier-Salaun MC, Van Milgen J, Montagne L. Feed restriction applied after weaning has different effects on pig performance and health depending on the sanitary conditions. J Anim Sci 2012; 90 (13): 4866-4875.

4. Bray GA: Obesity: historical development of scientific and cultural ideas. Int J Obes 909-926, 1990; 14 (11): 909-926.

5. Helander HF, Fandriks L. Surface area of the digestive tract - revisited. Scand J Gastroenterol 2014; 49 (6): 681-689.

6. Trbojevic-Stankovic JB, Milicevic NM, Milosevic DP et al. Morphometric study of healthy jejunal and ileal mucosa in adult and aged subjects. Histol Histopathol 2010; 25 (2): 153-158.

7. Tumova E, Zita L, Skrivanova V, Fucikova A, Skrivan M, Buresova M. Digestibility of nutrients, organ development and blood picture in restricted and ad libitum fed broiler rabbits. Arch Geflügelk 2007; 71 (1): S6-S12.

8. Fernandez-Carmona J, Blas E, Pascual JJ, Maertens L, Gidenne T, Xiccato G, Garcia J. Recommendations and guidelines for applied nutrition experiments in rabbits. World Rabbit Sci 2005; 13 (4): 209-228.

9. SAS Institute Inc. The SAS System for Windows. Release 9.1.3., SAS Institute Inc., Cary, NC, 2003.

10. Alencar AC, Neves RH, de Oliveira AV, Machado-Silva JR. Changes in the small intestine of Schistosoma mansoni-infected mice fed a high-fat diet. Parasitology 2012; 139 (6): 716-725.

11. de Oliveira Belem M, Cirilo CP, de Santi-Rampazzo AP, Schoffen JP, Comar JF, Natali MR, de Almeida Araujo EJ. Intestinal morphology adjustments caused by dietary restriction improves the nutritional status during the aging process of rats. Exp Gerontol 2015; 69 (1): 85-93.

12. Fassbinder-Orth CA, Karasov WH. Effects of feed restriction and realimentation on digestive and immune function in the Leghorn chick. Poult Sci 2006; 85 (8): 1449-1456.

13. Genton L, Cani PD, Schrenzel J. Alterations of gut barrier and gut microbiota in food restriction, food deprivation and protein-energy wasting. Clin Nutr 2015; 34 (3): 341-349.

14. Tsiouris V, Georgopoulou I, Batzios C, Pappaioannou N, Ducatelle $\mathbf{R}$, Fortomaris $\mathbf{P}$. Temporary feed restriction partially protect broilers from necrotic enteritis. Avian Pathol 2014; 43: (2): 139-145.

15. Knudsen C, Combes S, Briens C et al. Quantitative feed restriction rather than caloric restriction modulates the immune response of growing rabbits. J Nutr 2015; 145 (3): 483-489.

16. Lalles JP, David JC. Fasting and refeeding modulate the expression of sress proteins along the gastrointestinal tract of weaned pigs. J Anim Physiol Anim Nutr (Berlin) 2011; 95 (4): 478-488.
17. Tumova E, Volek Z, Chodova D et al. The effect of 1-week feed restriction on performance, digestibility of nutrients and digestive system development in the growing rabbit. Animal 2016; 10 (1): 1-9.

18. de Oliveira MC, da Silva DM, Borgel Dias DM. Effects of feed restriction on organs and intestinal mucosa of growing rabbits. Rev Bras Zootec 2013; 42 (7): 530-534.

19. Yakubu A, Salako AE, Ladokun AO, Adua MM, Bature TUK. Effects of feed restriction on performance, carcass yield, relative organ weights and some linear body measurements of weaner rabbits. Pak J Nutr 2007; 6 (4): 391-696.

20. Wijtten PJ, Hangoor E, Sparla JK, Verstegen MW. Dietary amino acid levels and feed restriction affect small intestinal development, mortality, and weight gain of male broilers. Poult Sci 2010; 89 (7): 1424-1439.

21. Tumova E, Volek Z, Makovicky P, Chodova D. Effects of a one week feed restriction in the growing rabbit : Part 2 - Development of the digestive system. Proceedings of the 10th World Rabbit Congress. Sharm El-Sheikh, Egypt. 2012, 621-624.

22. Makovicky P, Tumova E, Volek Z, Makovicky P, Vodicka P. Histological aspects of the small intestine under variable feed restriction: The effect of short and intensive restriction on a growing rabbit model. Exp Ther Med 2014; 8 (5): 1623-1627.

23. Laroque P, Keenan KP, Soper KA, Dorian C, Gerin G, Hoe CM, Duprat P. Effect of early body weight and moderate dietary restriction on the survival of the Sprague-Dawley rat. Exp Toxicol Pathol 1997; 49 (6): 459-465.

24. Moraal M, Leenaars PP, Arnts H, Smeets K, Savenije BS, Curfs JH, Ritskes-Hoitinga M. The influence of food restriction versus ad libitum feeding of choe and purified diets on variation in body weight, growth and physiology of female Wistar rats. Lab Anim 2012; 46 (2): 101-107.

25. Johnstone A. Fasting for weight loos: an effective strategy or latest dieting trend? Int J Obes (London) 2015; 39 (5): 727-733.

26. Zhang JG, Sun XW, Gao P, Xie L, Liu WQ, Xiao HT, Ai H. Food restriction alters villi morphology in obese rats: gut mechanism for weight regain? Exp Biol Med (Maywood) 2012; 237 (9): 993-999.

27. Dou Z, Gregersen S, Yhao J, Yhuang F, Gregersen H. Effect of refeeding after starvation on biochemical properties in rat small intestine. Med Eng Phys 2001; 23 (8): 557-566.

28. Wojciak RW. Effect of short-term food restriction on iron metabolism, relative well-being and depression symptoms in healthy women. Eat Weight Disord 2014; 19 (3): 321-327.

29. Grigg M, Bowman J, Redman S. Disordered eating and unhealthy weight reduction practises among adolescent females. Prev Med 1996; 25 (6): 748-756.

30. Neumark-Sztainer D, Wall M, Larson NI, Eisenberg ME, Loth K. Dieting and disordered eating behaviors from adolescence to young adulthood: findings from a 10-year longitudinal study. J Am Diet Assoc 2011; 111 (7): 1004-1011. 